\title{
Journal of Robotics and Mechanical Engineering Research
}

\section{Analysis and Measurement of Human Motion: Modern Protocols and Clinical Considerations}

\author{
Andrea Ancillao \\ Department of Mechanical and Aerospace Engineering, Sapienza University of Rome, Italy
}

\begin{abstract}
*Corresponding author: Andrea Ancillao, Department of Mechanical and Aerospace Engineering, Sapienza University of Rome, Via Eudossiana 18, 00183, Roma, Italy; Tel: 0644585585; Email: andrea.ancillao@hotmail.com
\end{abstract}

Article Type: Research, Submission Date: 19 October 2016, Accepted Date: 8 November 2016, Published Date: 15 December 2016.

Citation: Andrea Ancillao (2016) Analysis and Measurement of Human Motion: Modern Protocols and Clinical Considerations. J Robot Mech Eng Resr 1(4): 30-37.

Copyright: (C) 2016 Andrea Ancillao, et al. This is an open-access article distributed under the terms of the Creative Commons Attribution License, which permits unrestricted use, distribution, and reproduction in any medium, provided the original author and source are credited.

\section{Abstract}

The study of movement has always fascinated artists, photographers and researchers. Across the years, several attempts to capture, freeze, study and reproduce motion were made. Nowadays, the possibility to have quantitative data may support the diagnosis and treatment of many pathologies. Thus, in many rehabilitation facilities, a motion analysis laboratory is considered a must-have for diagnosis and patient's monitoring. This paper reviews the first historical attempts, based on photography, and their earliest results, till the state-of-the-art technology used today, i.e. the optoelectronic system. Working principle of the optoelectronic system is described as well as its application and setup in the clinical practice. Modern functional evaluation protocols are reviewed, with pro and cons and examples of clinical results and discoveries. Results of measurements conducted on subjects with neuro-motor pathologies are reviewed. Special attention is paid to the most common motion analysis exam that is nowadays worldwide standardized, i.e. the Gait Analysis. Examples of Gait Analysis on subjects with pathology and follow-up are reviewed.

Keywords: Biomechanics, Functional evaluation, Measurements, Medical devices, Optoelectronic System, Rehabilitation.

Abbreviations: OS: Optoelectronic System; GA: Gait Analysis; MoCap: Motion Capture; CP: Cerebral Palsy.

\section{History of Motion Analysis}

The study of principles movement, human and animal, is an intriguing topic that has always fascinated the curiosity of researchers. In fact, across the years, many studies and publications were made, trying to figure out the principles of movement and its biomechanical causes and effects.

Human/animal biomechanics involves multiple anatomical systems (nervous, muscular, visual, auditory etc.) and a strong coordination between the systems and the limbs, resulting in smooth and elegant movements, which may be very simple in the effect, but very complex from the mechanical point of view. Examples range from the animal quadrupedal walking or human bipedal walking (maintaining balance), to the most extreme sport performances.

For years, Engineers tried to reproduce natural movement strategies in mechanical machines or robots, but no one, has ever been successful in equalling such complex, smooth and beautiful motor performance.

A close attempt was the one achieved by the Dutch artist Theo Jansen that created some kinetic sculptures, named Strand beest (that means Beach Beasts). This sculptures were made of PVC, wood, and fabric airfoils that collect the power given by the wind. By means of a mechanical design, wind power is stored in flywheels and transferred to several legs that move sequentially and achieve a walking effect (Figure 1). These beasts are able to reproduce a multi-legged walking pattern and are free to walk around by themselves (http://www.strandbeest.com).

Modern examples of devices designed to emulate and support human motion are robotic devices and orthosis. E.g. some advanced mechanisms were designed in the form of an exoskeleton to assist people with hand-motion impairments, by reproducing the physiological kinematics of fingers [1]. The key point in the design of such mechanism is that, while the exoskeleton should

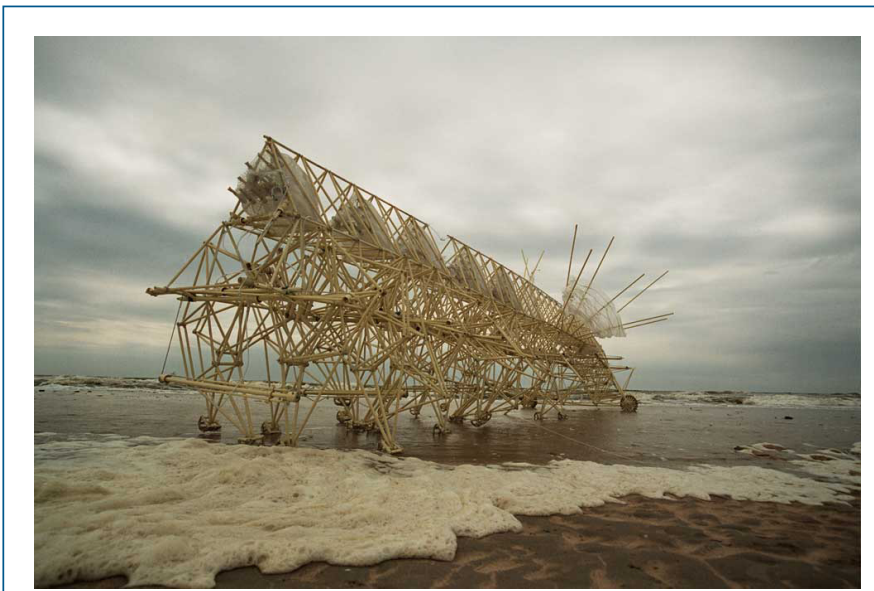

Figure 1: A Strandbeest designedby Theo Jansen. (http://www. strandbeest.com) 
accurately reproduce the natural biomechanics, it should be small, portable and cost-effective as well [2].

Other examples are the robotic devices designed to simulate the biomechanics of human breathing $[3,4]$ and to assist human walking in rehabilitation programs [5]. The design of such robotic devices requires the parallel development of advanced mathematical algorithms for generating inverse kinematics maps [6].

It is clear that studying human/animal biomechanics represents a big research challenge, aimed to understand and reproduce the principles of movement and to finally answer the question: how can that perfect machine, that is human body, achieve such smooth movements in a so simple and natural way?

The first biomechanical studies were conducted by means of photography techniques, where an effective representation of motion could be obtained by taking sequential pictures at a fixed time interval. This technique was known as chronophotography. The most known attempts were the works of the English photographer Eadweard Muybridge (9 April 1830 - 8 May 1904, Figure 2). He used advanced photographic instrumentation (for that time) to take sequential photography of animals and people performing motor tasks. His works were appreciated for their artistic and scientific value. His most famous work, The Horse in Motion (Figure 3), was inspired by a biomechanical question that was popular in that period: is there a moment in which all the four feet of a trotting horse are off the ground at the same time? Till that time, in fact, most artists used to paint horses with one foot always on the ground. The question was intriguing, because the forward movement logically required a moment of complete loss of contact with the soil, but no one had ever observed it, as the human eye is not fast enough to catch the moment.

To answer that question, Muybridge took sequential shoots of

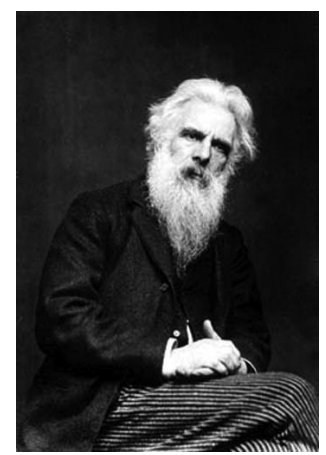

Figure 2: Eadweard Muybridge

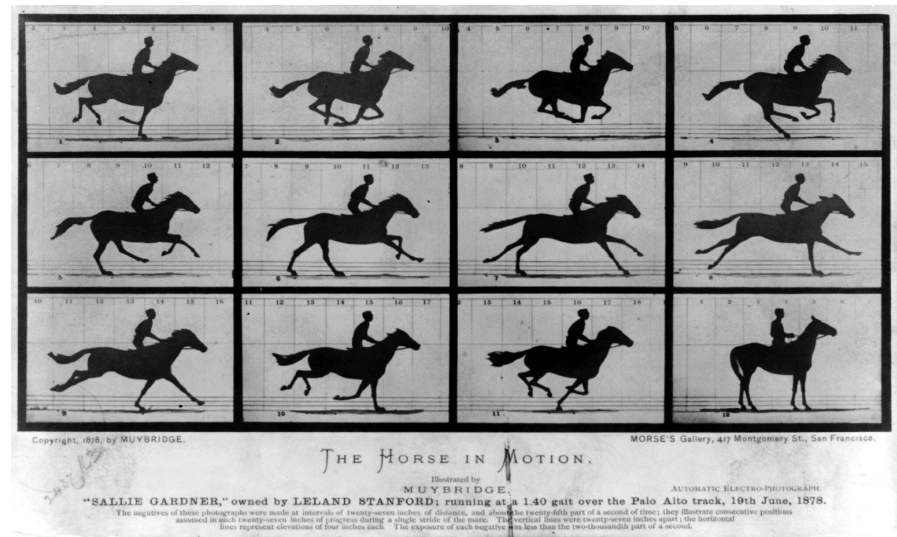

Figure 3: The Horse in Motion, by E. Muybridge, 1878 a galloping horse using an array of 12 cameras placed along a racetrack [7]. The images clearly showed that there was a time in the running stride when the horse did actually have all the four hooves off the ground (Figure $3,1^{\text {st }}$ line, $3^{\text {rd }}$ image).

Other famous Muybridge's works are "The Woman Walking Downstairs" and "Two women kissing", shown in Figures 4 and 5. In these works the author used the chronophotography technique to represent the motion of human action and to provide an objective evaluation of the posture and motor strategy involved in the action. The "Woman Walking Downstairs" is a remarkable piece of work as it considered one of the first attempts to objectively study the biomechanics of the human body while performing everyday tasks. The "Two women kissing" is considered the first ever filmed kiss.

In 1882, the French scientist and photographer Étienne-Jules Marey (5 March 1830, 15 May 1904) invented a device capable of taking 12 consecutive frames in a second. This device was a shotgun modified to capture light on a photo-sensitive disc that collected the 12 frames consecutively. The device was named chronophotographic gun (Figures 6,7) and the resulting pictures in motion can be considered the precursor of the cinematographer, invented by Lumière brothers in 1895 .

Marey's studies were mainly focussed on animals (horses, birds, cats and others) and human locomotion as well [8]. The most famous work is the movie that demonstrates how falling cats always land on their feet (Figure 8). He also conducted very similar studies on chickens and dogs finding out that they could do almost the same [9]. Marey also conducted studies on the biomechanics of human walking (Figure 9) and subsequently improved the photographic technique by adding markers on the subject's body. This markers resulted in bright dots or lines on

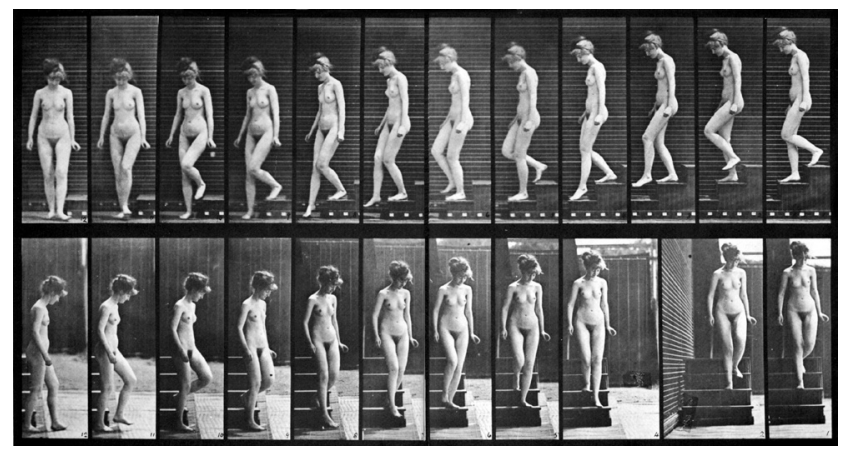

Figure 4: Woman Walking Downstairs, by E. Muybridge, 1887

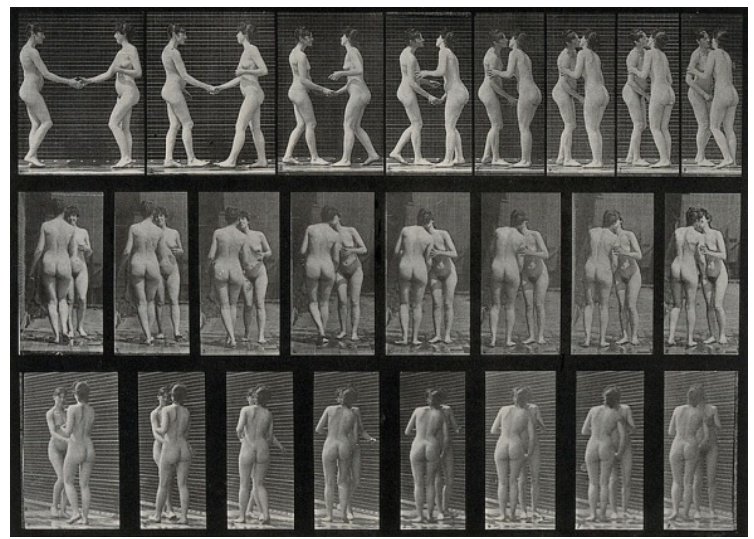

Figure 5: Two women kissing, by E. Muybridge, 1887 
the developed film, allowing an accurate identification of body segments and landmarks and tracking their position over time (Figure 10).

Following years brought several advances in photographic techniques and cinematography that allowed the biomechanical analysis to become more accurate and detailed. The possibility to obtain an objective evaluation of human motor performance has captured the interest of clinicians and today it is considered a valid method to study motor performance and to support

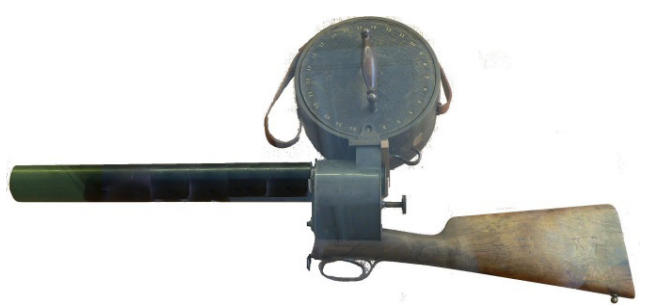

Figure 6: The Chronophotographic Gun, by Étienne-Jules Marey

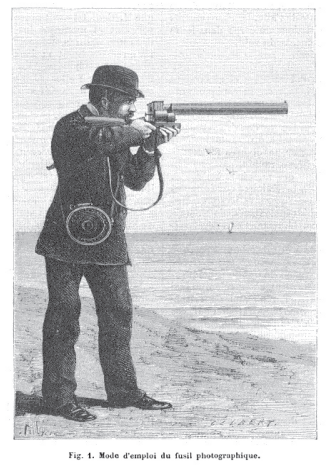

Figure 7: Chronophotographic Gun in use

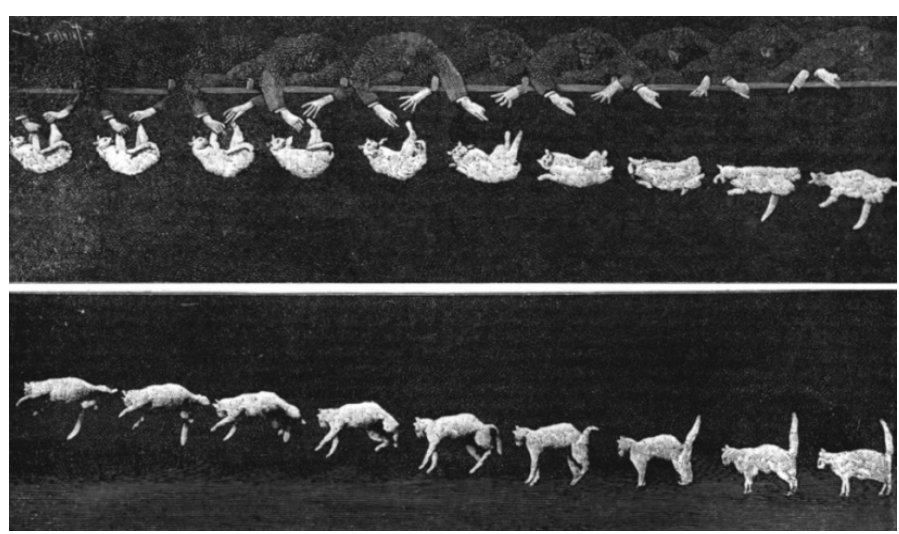

Figure 8: Falling cat, by Étienne-Jules Marey, 1894

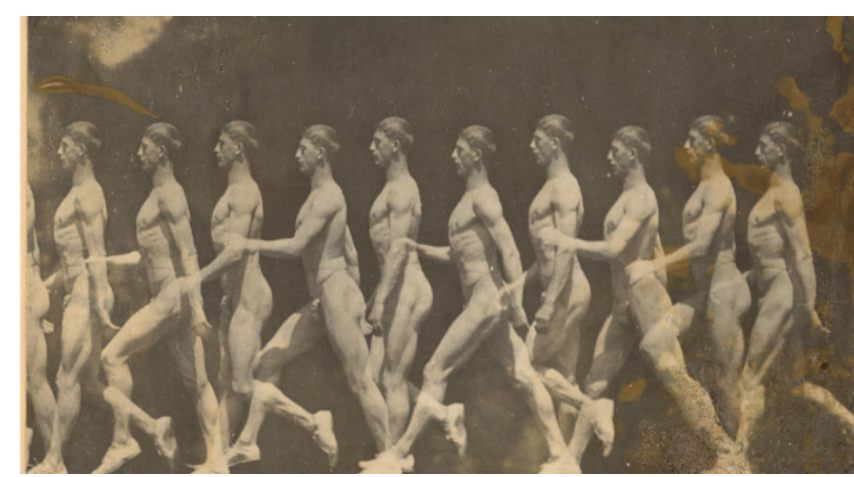

Figure 9: Man Walking, by Étienne-Jules Marey, 1890s the diagnosis with quantitative data [10]. Thus, biomechanical analysis has switched from a purely academic discipline to a powerful clinical tool for functional evaluation and diagnosis of motor disorders. It was proved of being useful in supporting the diagnosis of motor disorders and treatment follow-up by providing quantitative information [11-16].

\section{Modern Functional Evaluation}

The process of recording and reconstructing the movements of a subject, actor, animal or any moving object, is nowadays known as motion capture, or MoCap for short.

Nowadays, the gold standard method for MoCap is the use of an Optoelectronic System (OS) [17], that is widely employed in cinema and video games industry, for the modelling of humanoid characters, and in medicine/sport science, for the functional evaluation of patients and athletes.

OSs use several infra-red cameras, placed along the perimeter of a laboratory, to observe the scene from different angles. Each camera has a IR strobe coaxial to the lens that lightens up the field of view. Light produced by the strobes bounces on the reflective markers that are placed on anatomical landmarks over the subject/object to acquire. Camera's sensors have a filter that allows them to collect only the infra-red radiation scattered by the markers, while discarding the ambient light. This allows to obtain high contrast images of the markers that are seen as white dots on a black background. A further method to discard ambient light and to reduce artefacts (false marker detection due to reflecting objects in the room), is to drive the strobes at a specified frequency (flashing).

The two-dimensional images acquired by cameras are mixed by means of stereo-photogrammetry techniques that allows to reconstruct the three-dimensional coordinates of markers, that in turn shape the biomechanical model of the subject (Figure 11).

For each marker the sensor detects a white dot, whose diameter depends on the size of the marker itself, resolution of the sensor and the distance between the marker and the camera. The centroid of the white area is computed in order to define the marker positions with respect to the camera's frame (Figure 12).

By means of parallax algorithms it is possible to reconstruct the $x, y, z$ Cartesian coordinates of each marker in a $3 \mathrm{D}$ virtual space. The coordinates are acquired as time series representing the duration of motion.

For the algorithm to work, each marker should be seen by at

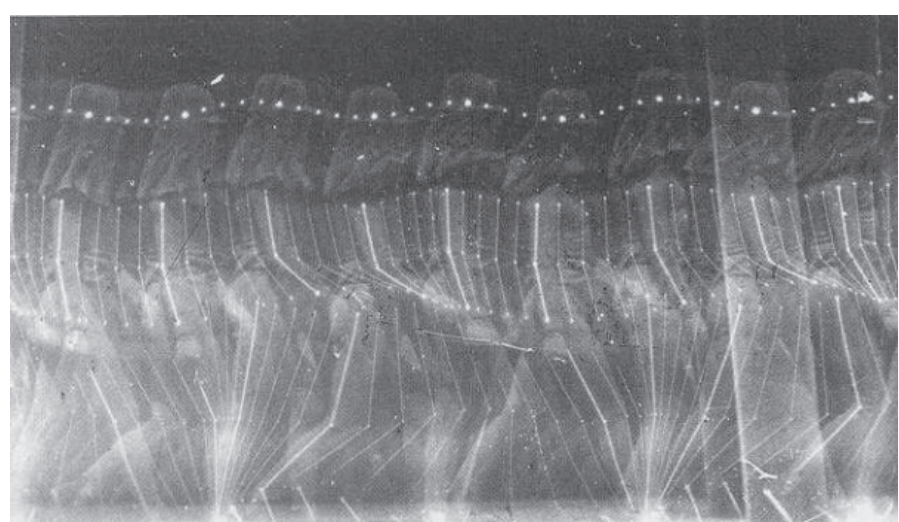

Figure 10: Walk, by Etienne-Jules Marey, 1886 


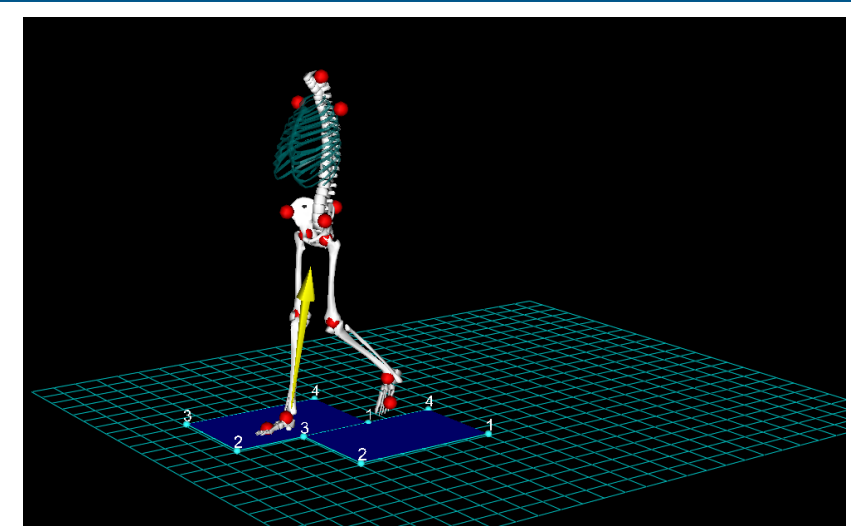

Figure 11: Biomechanical model of a walking subject. The model is reconstructed by using the information from the Optoelectronic System and the force plates

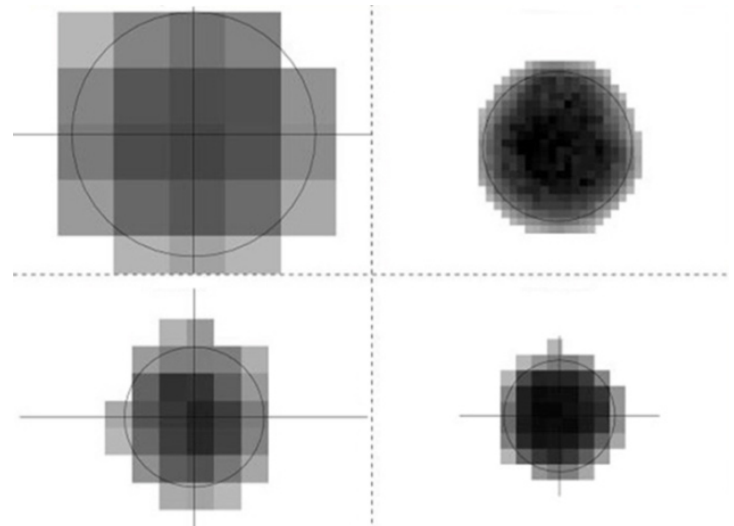

Figure 12: Markers seen by camera's sensor and centroid estimation at different resolutions.

least two cameras. As the markers may be easily hidden during motion, many cameras are placed along the perimeter of the room, to ensure that at least two cameras see each marker during the recording. If more than two cameras see the same marker, redundancy can be used to optimize, and therefore increase, the accuracy of the reconstruction [17-20].

The commonly used markers are small spheres or hemispheres with IR-reflecting external surface. The most common diameters are $10 \mathrm{~mm}, 5 \mathrm{~mm}, 3 \mathrm{~mm}$ and $1 \mathrm{~mm}$ (Figure 13).

The accuracy of the system strongly depends on the resolution of cameras' sensor and the volume of the laboratory. A common

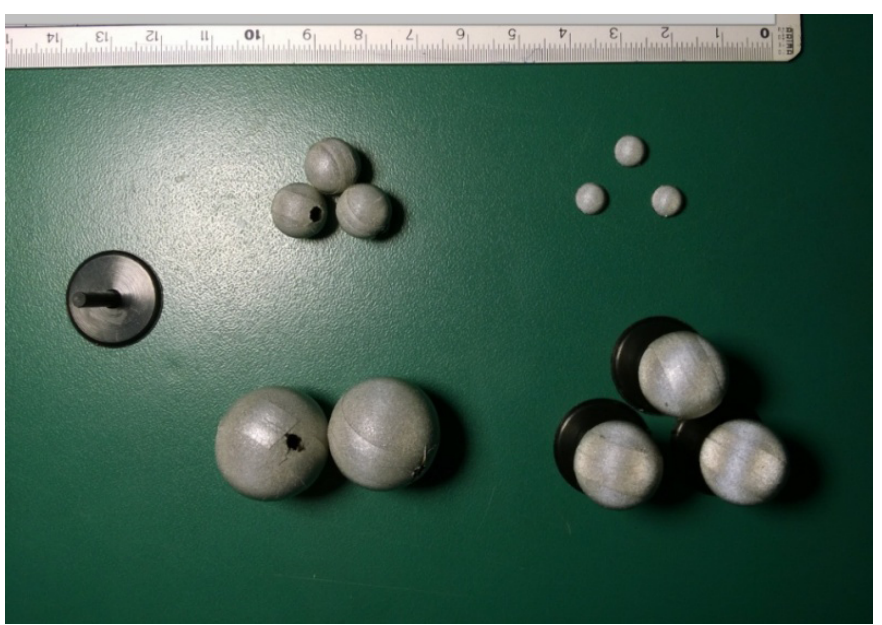

Figure 13: IR reflectivemarkersfor motion capture and support for application on subject's skin. movement analysis laboratory consists in a physiotherapy gym with an useable space of about $100 \mathrm{~m}^{2}$. To such a volume, corresponds an accuracy of $\sim 1 \mathrm{~mm}$ on the reconstruction of $x, y, z$ coordinates.

The Optoelectronic System can also record real time data streamed from other instrumentation, such as force platforms, electromyography and video recording system, and synchronize it to the kinematic recording [21]. This enables a multifactorial and multivariate analysis of motion.

The key point in motion capture is the reconstruction of a subject model from the markers coordinates (Figure 11). To obtain this, markers have to be placed on specific anatomical landmarks, according to a protocol which depends on the anatomical district or on the task that needs to be investigated.

The design of a functional evaluation protocol is not an easy task, as limbs have to be modelled as rigid segments reconstructedfrom the physical markers that should berigidly fixed over the skin of the subject. The modelling therefore runs under the rigid body assumption, which means that the modelled limb does not undergo modifications during the motion. Under this assumption, at least three markers are required to track each segment [17].

It is clear that the tracking of rigid bodies is affected by artefacts/ errors due to wrong positioning, skin sliding over the bone, etc. This errors are usually addressed as "soft tissue artefacts" [20]. These effects can be reduced by marker redundancy, accurate marker placement and accurate definition of anatomical landmark by the design of an ad-hoc functional evaluation protocol.

The general guidelines for markers placement identify the most suitable landmarks as the point where the effect of the skin sliding over the bones is minimum. This landmarks are often identified on bone prominences, points of reference for joint rotations (elbow, knee, ankle, etc.) and limb extremities (toe, fingers, etc.).

\section{Functional evaluation protocols}

Many functional evaluation protocols were developed across the years, depending on tasks to investigate. Testing ranged from general motor abilities, to high level neuro-motor coordination, that reflect the brain's ability to conceive, organize, program and carry out a sequence of actions [22].

The most known functional evaluation protocol requiring MoCap is the Gait Analysis (GA), i.e. the quantitative study of the walking strategy of a subject and its implications on the posture. GA provides detailed quantitative information about the functionality of the anatomical districts directly involved in the act of walking: foot, ankle, knee, hip and pelvis.

During a GA, the subject is asked to walk along a pathway within the lab, while a biomechanical model is reconstructed (Figure 11).

GA allows the estimation of: (i) kinematic parameters, the anatomical angles and their variation across three anatomical reference planes (sagittal, coronal and horizontal); (ii) spatiotemporal parameters: cadence, velocity, step length, stance time, stride time, asymmetry in stride, etc.; (iii) kinetic parameters: ground reaction forces in three dimensions and their evolution over time; (iv) EMG tracks and other analog tracks describing muscular activation, timings and strength. 
Several marker protocols were proposed for the recording of a GA [23]. Some examples are the CAST [24] and the Davis protocol [25] that are widely used in today's clinical practice.

Nowadays GA is widely recognized as a multifactorial and powerful clinical tool $[10,26]$.

GA was already applied to the characterization of the walking strategy in: Parkinson's disease [12], Down Syndrome [27], Ehlers-Danlos Syndrome [28], Cerebral Palsy (CP) [26,29] and it was used to validate the effects of novel treatments in subjects with neurological disorders [12,13,30,31].

Clinical decisions, rehabilitative treatments and follow-up evaluation are often based on the results of GA exams [10,32], especially in the case of $\mathrm{CP}$ and spastic paresis that may induce motor disorders at different levels. Actually, very different gait patterns are observed in patients with CP [31,33,34]. Some examples of gait abnormalities observed in $\mathrm{CP}$ patients are: the equinus gait pattern, that involves alteration of ankle joint functionality [35]; crouch gait, that involves abnormal knee flexion [29]; and pelvis abnormal anti-retroversion with overall range of motion limitation due to spasticity [29].

As further examples, GA was used to monitor the long term effects of orthopaedic surgery on children with $\mathrm{CP}$, quantifying the improvements over the time [36]. Patients with Prader-Willi syndrome [30] were found to have a significant improvement in GA after an osteopathic treatment. Sale et al. [12] used GA to measure gait improvements in subjects with Parkinson's Disease undergoing a robotic treatment. Finally, a treatment of neuromuscular taping improved gait of a subject with Joint Hypermobility Syndrome [13].

Several research works were aimed at the design and validation of MoCap protocols for the functional evaluation of some anatomical districts or functional tasks. Examples follow.

Ancillao et al. [16] designed a protocol for the analysis of displacements of human mandible. It was based on 12 small markers placed on the face of the subject that allowed the measurements of small displacement of the jaw and the head. The protocol was applied to subjects with Ehlers-Danlos syndrome and allowed a quantitative analysis of jaw dislocation and its effects on posture on subjects with pathology. The same authors designed a protocol for the evaluation of facial movements and expressions [37]. It was based on a total of 20 markers placed on the face, head and upper body of the subject (Figure 14). The high accuracy of the protocol allowed to measure the effects of stroke on facial conformation and mobility, giving a substantial help for the diagnosis and follow up of such pathologies [37]. Ancillao, et al. also designed a protocol for the analysis of handwriting by means of the OS [38]. This protocol was based on 9 markers of $5 \mathrm{~mm}$ diameter placed on the cap of a common pen/pencil and on the corners of an A4 sheet and 12 marker on the upper limb and head of the subject (Figure 15).

The drawing protocol was proved to be able to reconstruct handwriting with an accuracy of $\sim 0.6 \mathrm{~mm}$. This method allowed to produce quantitative data supporting many clinical tests that are commonly administered by the Pen-and-Sheet method. For these tests, a score is qualitatively assigned by a trained operator [38]. Some examples are the Denver Developmental Screening

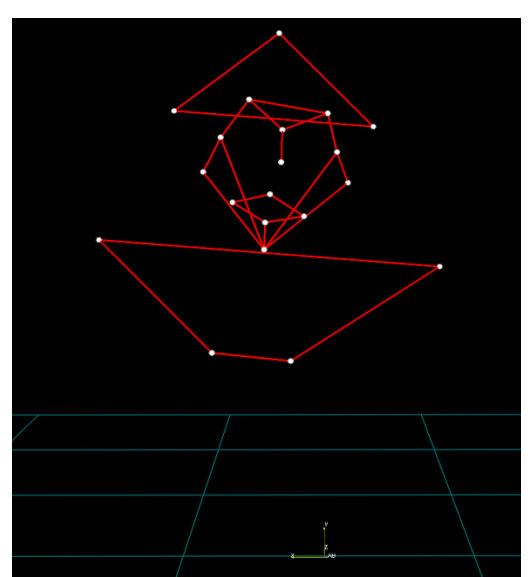

Figure 14: Marker protocol designed by Ancillao et al. [37] in use for the reconstruction of facial expressions

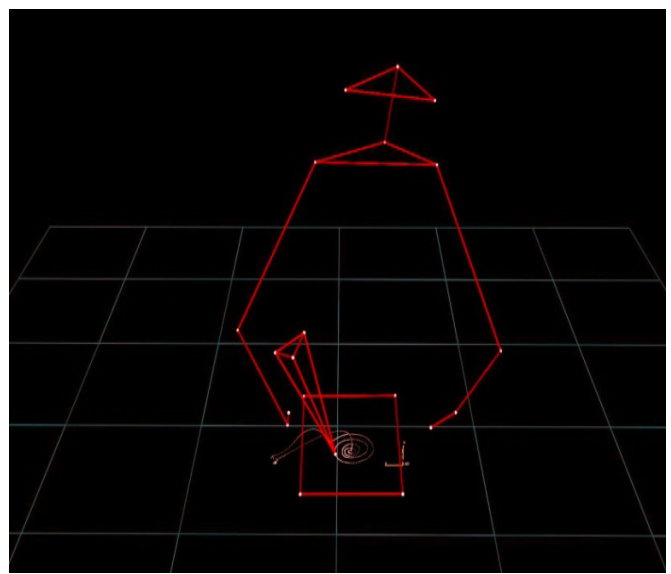

Figure 15: Marker protocol designed by Ancillao et al. [38] in use for the reconstruction of drawing.

Test, in which the subject is asked to draw simple geometrical figures, such as a circle, a square and a triangle $[39,40]$ and the Clock Drawing Test, in which the subject is asked to draw a clock indicating current time [41,42]. The MoCap protocol [38] allows the estimation of velocity, starting/ending points, dimensions and length of the track, angles, etc. that play animportant role in the diagnosis and follow-up evaluation of neurological diseases, such as Parkinson's disease [43,44], Multiple Sclerosis [45], or developmental disabilities [39,46,47].

The use of the OS is preferable to graphics tablets because it works with a regular pen and sheet without altering the writing conditions by inserting a layer between the sheet and the desk [38]. Moreover, the OS provides additional information that is not recorded by the graphic tablet, i.e. the trajectory and speed of the pen tip while it is not in contact with the paper, the orientation of the pen in space, etc. Measures of angles and motion of anatomical joints (finger, wrist, elbow, shoulder) and general posture of the subject may also be recorded at the time of the writing $[38,40]$. The drawing protocol [38] was applied to the study of drawings performed by subjects with Down Syndrome [48] that were asked to draw simple geometrical shapes, according to the Denver screening test [39]. Handwritten tracks, as well as motion of the arm and posture, were recorded by means of the OS. The study demonstrated that Down Syndrome subjects draw faster than controls but with less accuracy [48]. 
Another protocol for the functional evaluation of the upper limb was designed by Vimercati, et al [49] to investigate the strategy chosen by subjects with Down Syndrome that were asked to sequentially hit some targets by a stick hold in hand while sitting in front of a table. The protocol was composed of 12 passive markers that provided a fine reconstruction of the motor strategy chosen by the subjects with Down Syndrome that are known to have poor coordination, high rates of failure and slower reaction time, mainly due to lower muscle tone and ligament laxity [50]. Linear and angular velocities were measured, as well as acceleration, showing in agreement with other studies $[51,52]$ that children with Down Syndrome moved slower and with reduced peak velocity than normally developed children. The motion of the upper limb was also studied in a patient with hemiplegia that was treated with neuromuscular taping [53]. The subject, while sitting in front of a table, was asked to reach a target with her finger on the affected arm and then bring the arm back to the resting position. The markers were placed on the head, the trunk, the arm, forearm and hand and the reaching movement was segmented into three sequential phases. The OS allowed to compute timings of the phases, smoothness of the movement, joint ranges of motion and reaching accuracy. The analysis was repeated "pre" and "post" treatment and it was proved that motion in the "post" session was smoother, faster and less segmented [53].

A detailed study on reaching is the one by Butler, et al [54] that studied the ability of children with cerebral palsy to reach, grasp and transport objects, compared to a control group. More in details, children were asked to reach a cup of water, bring it to the mouth and simulate water drinking. The motion was studied by an OS and a marker protocol on the upper limb, composed of 17 markers. The parameters measured were: trunk flexion/ extension and rotation, shoulder elevation, elbow flexion/ extension, forearm pronation/supination and wrist flexion/ extension. The study showed that children with cerebral palsy had reduced elbow extension followed by increased wrist flexion and trunk motion [54].

The motion analysis of the upper limb is crucial to study the biomechanical activities that involve fast and accurate movements such as bowing to play string instruments. In the work of Turner-Stokes and Reid [55], the authors developed a protocol to study the motion of the bowing arm of musicians. The protocol involved an OS, reflective markers placed on the bowing arm and it was aimed to the diagnosis of neuro-motor diseases that are common among professional musicians [55]. The authors compared motor strategies trajectories adopted by players of different bowing instruments from cello to violin. Quantitative results were proven reproducible and demonstrated clear differences between the instruments as well as stylistic differences between the players. The range of motion of the shoulder correlated with the type of the instrument, being larger on the cello and smaller in the case of the violin. Instead, the range of motion of the elbow was greater on the violin [55]. The high range of motion of the shoulder was correlated to the neck and shoulder symptoms that are common among cellists.
Another study on interlimb coordination in violin players was conducted by Baader, et al [56]. The use of OS allowed recording finger trajectories and bowing motion providing quantitative results in terms of velocity and timings. Anticipatory mechanisms in finger-press, synchronization, and errors in timings were exploited, proving that the optoelectronic measurements are accurate and powerful in assessing small, fast and accurate movements.

Biomechanical analysis becomes critical when modelling small anatomical segments such as fingers. Some study were conducted about the definition and validation of biomechanical models of thumbs and wrist joints [57-61]. Cerveri, et al [58] validated a kinematic model of the trapezio-metacarpal joint. The motion was reconstructed by nine passive markers applied on the surface of hand and fingers, and then acquired through an OS. The model allowed representing motion of the thumb joint across the three anatomical axes. Analysis of inaccuracies showed that the model was able to reconstruct kinematics with an error of $5 \mathrm{~mm}$ for linear distances and $6^{\circ}$ for angles. Small, et al [59] modelled and measured motion of the wrist by means of an OS and stereoradiography. The detailed kinematic analysis was performed to extract Euler angles among the anatomical planes. Angular uncertainties due to the OS were estimated about $6 \mathrm{~mm}$ in accordance with the results of Cerveri, et al [58]. Results showed that measurements conducted by OS and by stereo-radiography were comparable with similar accuracies [59]. Opposite to stereoradiography, OS provides a non-invasive method for accurate biomechanical analysis of the wrist joint [59].

\section{Conclusion}

The capture of motion has always fascinated the artists the photographers and, nowadays, the researchers.

Across the years several technologies were developed, allowing a more accurate quantitative reconstruction of motion.

Today the gold standard for motion capture is the optoelectronic system, whose use is widespread in the motion picture and video games industry, for the modelling of humanoid characters, as well as in medicine/sport science, for the functional evaluation of patients and athletes.

Several functional evaluation protocols, involving the OS, have been developed and validated, allowing an accurate evaluation of many anatomical districts and motor tasks. This allowed a deep investigation of the motor features of subject with pathologies involving the nervous system and the musculoskeletal system.

The possibility to have non-invasive procedures providing quantitative data to document the evolution or improvement of a pathology, is extremely useful to support the diagnosis and treatment of such pathologies. In many clinical rehabilitation facilities, motion analysis and Gait Analysis are considered a must-have for diagnosis and patient's monitoring.

In the next years, accuracy of the systems will improve, allowing an even more accurate analysis, and therefore more possibilities for biomechanical analysis. 
Citation: Andrea Ancillao (2016) Analysis and Measurement of Human Motion: Modern Protocols and Clinical Considerations. J Robot Mech Eng Resr 1(4): 30-37.

\section{References}

1. Conti R, Meli E, Ridolfi A. A novel kinematic architecture for portable hand exoskeletons. Mechatronics. 2016; 35:192-207.

2. Conti R, Allotta B, Meli E, Ridolfi A. Development, design and validation of an assistive device for hand disabilities based on an innovative mechanism. Robotica. 2016.

3. Massaroni C, De Leo F, Schena E, Saccomandi P, Silvestri S. Metrological properties evaluation of a chest wall simulator during simulated quiet breathing. In MeMeA2015; 2015. p. 600-605.

4. Massaroni C, Schena E, Bastianini F, Scorza A, Saccomandi P, Lupi $G$, et al. Development Of A Bio-Inspired Mechatronic Chest Wall Simulator For Evaluating The Performances Of Opto-Electronic PIethysmography. Open Biomed. Eng. J. 2014; 8(1):120-130.

5. Sale P, De Pandis MF, Galli M, Cimolin V, Stocchi F, Ancillao A, et al. Recovery of walking performance with robot assisted end effector gait trainer in people with Parkinson disease: Preliminary report. Gait Posture. 2013; 37(2013):S15-S16.

6. Mayyas M, Mellish R. A method for the automatic generation of inverse kinematic maps in modular robotic systems. Int. J. Adv. Robot. Syst. 2016; 13(5):1-15.

7. Muybridge E. The science of the horse's motions. Sci. Am. 1878; 39:241.

8. Marey E. Terrestrial and Aerial Locomotion. vol. 1. New York: Appleton and company; 1874.

9. Marey M. Des mouvements que certains animaux exécutent pour retomber sur leurs pieds, lorsqu'ils sont précipités d'un lieu élevé. Acad. Sci. 1894; 119:714-717.

10. Whittle M. Clinical gait analysis: A review. Hum. Mov. Sci. 1996; 15(3):369-387.

11. Sale P, De Pandis M, Vimercati S, Sova I, Foti I, Tenore N, et al. The relation between Parkinson's disease and ageing-Comparison of the gait patterns of young Parkinson's disease subjects with healthy elderly subjects. Eur. J. Phys. Rehabil. Med. 2012; 48:1-7.

12. Sale P, De Pandis MF, Stocchi F, Domenica LP, Sova I, Cimolin V, et al. Robot-assisted walking training for individuals with Parkinson's disease: a pilot randomized controlled trial. BMC Neurol. 2013; 13(1):50.

13. Camerota F, Galli M, Cimolin V, Celletti C, Ancillao A, Blow D, et al. The effects of neuromuscular taping on gait walking strategy in a patient with joint hypermobility syndrome/Ehlers-Danlos syndrome hypermobility type. Ther. Adv. Musculoskelet. Dis. 2015; 7(1):3-10.

14. Charbonnier C, Chagué S, Ponzoni M, Bernardoni M, Hoffmeyer P, Christofilopoulos P. Sexual activity after total hip arthroplasty: a motion capture study. J. Arthroplasty. 2014; 29(3):640-647.

15. Ancillao $A$, Patanè $F$, Rossi S, Pacilli A, Cappa P. Lower limb strength measurements by Hand Held Dynamometer assisted by optoelectronic system. In MMT2014; 2014. p. 1-2.

16. Ancillao A, Galli M, Celletti C, Castori M, Albertini G, Camerota F. Temporomandibular joint mobility in adult females with EhlersDanlos syndrome, hypermobility type (also known as joint hypermobility syndrome). J. Cranio-Maxillary Dis. 2012; 1(2):88-95.

17. Cappozzo A, Della Croce U, Leardini A, Chiari L. Human movement analysis using stereophotogrammetry. Part 1: theoretical background. Gait Posture. 2005; 21(2):186-196.
18. Chiari L, Della Croce U, Leardini A, Cappozzo A. Human movement analysis using stereophotogrammetry. Part 2: instrumental errors. Gait Posture. 2005; 21(2):197-211.

19. Della Croce U, Leardini A, Chiari L, Cappozzo A. Human movement analysis using stereophotogrammetry. Part 4: assessment of anatomical landmark misplacement and its effects on joint kinematics. Gait Posture. 2005; 21(2):226-237.

20. Leardini A, Chiari L, Della Croce U, Cappozzo A. Human movement analysis using stereophotogrammetry. Part 3. Soft tissue artifact assessment and compensation. Gait Posture. 2005; 21(2):212-225.

21. Ancillao A, Galli M, Rigoldi C, Albertini G. Linear correlation between fractal dimension of surface EMG signal from Rectus Femoris and height of vertical jump. Chaos, Solitons \& Fractals. 2014; 66:120-126.

22. Rosenbaum D. Human Motor Control. 2nd ed. Academic Press; 2009.

23. Ferrari A, Benedetti MG, Pavan E, Frigo C, Bettinelli D, Rabuffetti $\mathrm{M}$, et al. Quantitative comparison of five current protocols in gait analysis. Gait Posture. 2008; 28(2):207-216.

24. Cappozzo A, Cappello A, Della Croce U, Pensalfini F. Surface-marker cluster design criteria for 3-D bone movement reconstruction. IEEE Trans. Biomed. Eng. 1997; 44(12):1165-1174.

25. Davis RB, Õunpuu S, Tyburski D, Gage JR. A gait analysis data collection and reduction technique. Hum. Mov. Sci. 1991; 10(5):575587.

26. Carriero A, Zavatsky A, Stebbins J, Theologis T, Shefelbine SJ. Determination of gait patterns in children with spastic diplegic cerebral palsy using principal components. Gait Posture. 2009; 29(1):7175.

27. Galli M, Rigoldi C, Brunner R, Virji-Babul N, Giorgio A. Joint stiffness and gait pattern evaluation in children with Down syndrome. Gait Posture. 2008; 28:502-506.

28. Rigoldi C, Galli M, Cimolin V, Camerota F, Celletti C, Tenore N, et al. Gait strategy in patients with Ehlers-Danlos syndrome hypermobility type and Down syndrome. Res. Dev. Disabil. 2012; 33(5):14371442.

29. van den Noort J, Ferrari A, Cutti A, Becher J, Harlaar J. Gait analysis in children with cerebral palsy via inertial and magnetic sensors. Med Biol Eng Comput. 2013; 51(4):377-386.

30. Vismara L, Cimolin V, Galli M, Grugni G, Ancillao A, Capodaglio P. Osteopathic Manipulative Treatment improves gait pattern and posture in adult patients with Prader-Willi syndrome. Int. J. Osteopath. Med. 2016; 19:35-43.

31. Ancillao A, Van Der Krogt M, Buizer A, Witbreuk M, Cappa P, Harlaar $J$. Analysis of gait features variation pre and post SEML surgery in CP by means of GPS and MAP. Gait Posture. 2016; 49:120.

32. Assi A, Ghanem I, Lavaste F, Skalli W. Gait analysis in children and uncertainty assessment for Davis protocol and Gillette Gait Index. Gait Posture. 2009; 30(1):22-26.

33. Piccinini L, Cimolin V, D’Angelo MG, Turconi AC, Crivellini M, Galli M. 3D gait analysis in patients with hereditary spastic paraparesis and spastic diplegia: A kinematic, kinetic and EMG comparison. Eur. J. Paediatr. Neurol. 2011; 15(2):138-145.

34. Galli M, Cimolin V, Rigoldi C, Tenore N, Albertini G. Gait patterns in hemiplegic children with Cerebral Palsy: Comparison of right and left hemiplegia. Res. Dev. Disabil. 2010; 31(6):1340-1345. 
35. van der Krogt MM, Doorenbosch CAM, Becher JG, Harlaar J. Walking speed modifies spasticity effects in gastrocnemius and soleus in cerebral palsy gait. Clin. Biomech. 2009; 24(5):422-428.

36. Galli M, Cimolin V, Crivellini M, Albertini G. Long-term evaluation of isolated gastrocnemius fascia lengthening in children with cerebral palsy using gait analysis. J. Pediatr. Orthop. B. 2009; 18(5):228233.

37. Ancillao A, Galli M, Annese E, Criscuolo S, Vimercati SL, Le Pera D, et al. Quantitative evaluation of facial movements in adult patients with hemiplegia after stroke. Int. J. Signal Image Process Issues. 2016; 2016(1):1-10.

38. Ancillao A, Galli M, Vimercati SL, Albertini G. An optoelectronic based approach for handwriting capture. Comput. Methods Programs Biomed. 2013; 111(2):357-365.

39. Khalid PI, Yunus J, Adnan R, Harun M, Sudirman R, Mahmood NH. The use of graphic rules in grade one to help identify children at risk of handwriting difficulties. Res. Dev. Disabil. 2010; 31(6):16851693.

40. Galli M, Vimercati SL, Stella, Caiazzo G, Norveti F, Onnis F, et al. A new approach for the quantitative evaluation of drawings in children with learning disabilities. Res. Dev. Disabil. 2011; 32(3):10041010.

41. Aprahamian I, Martinelli JE, Neri AL, Yassuda MS. The accuracy of the Clock Drawing Test compared to that of standard screening tests for Alzheimer's disease: results from a study of Brazilian elderly with heterogeneous educational backgrounds. Int. Psychogeriatr. 2010; 22(1):64-71.

42. Brodaty $\mathrm{H}$, Moore $\mathrm{CM}$. The clock drawing test for dementia of the Alzheimer's type: A comparison of three scoring methods in a memory disorders clinic. Int. J. Geriatr. Psychiatry. 1997; 12(6):619-627.

43. De Pandis MF, Galli M, Vimercati S, Cimolin V, De Angelis MV, Albertini G. A new approach for the quantitative evaluation of the clock drawing test: preliminary results on subjects with Parkinson's disease. Neurol. Res. Int. 2010; 2010:283890.

44. Vimercati SL, Galli M, De Pandis MF, Ancillao A, Stella G, Albertini G. Quantitative evaluation of graphic gesture in subjects with Parkinson's disease and in children with learning disabilities. Gait Posture. 2012; 35:S23-S24.

45. Longstaff MG, Heath Ra. Spiral drawing performance as an indicator of fine motor function in people with multiple sclerosis. Hum. Mov. Sci. 2006; 25(4-5):474-491.

46. Casellato C, Zorzi G, Pedrocchi A, Ferrigno G, Nardocci N. Reaching and writing movements: sensitive and reliable tools to measure genetic dystonia in children. J. Child Neurol. 2011; 26(7):822-829.

47. Gilboa Y, Josman N, Fattal-Valevski A, Toledano-Alhadef H, Rosenblum $\mathrm{S}$. The handwriting performance of children with NF1. Res. Dev. Disabil. 2010; 31(4):929-935.
48. Vimercati SL, Galli M, Stella G, Caiazzo G, Ancillao A, Albertini G. Clumsiness in fine motor tasks: evidence from the quantitative drawing evaluation of children with Down Syndrome. J. Intellect. Disabil. Res. 2014:1-9.

49. Vimercati S, Galli M, Rigoldi C, Ancillao A, Albertini G. Motor strategies and motor programs during an arm tapping task in adults with Down Syndrome. Exp. Brain. 2013.

50. Morris AF, Vaughan SE, Vaccaro P. Measurements of neuromuscular tone and strength in Down's syndrome children. J. Ment. Defic. Res. 1982; 26(1):41-46.

51. von Hofsten C, Rönnqvist L. The structuring of neonatal arm movements. Child Dev. 1993; 64(4):1046-1057.

52. von Hofsten C. Structuring of Early Reaching Movements: A Longitudinal Study. J. Mot. Behav. 1991; 23(4):280-292.

53. Camerota F, Galli M, Cimolin V, Celletti C, Ancillao A, Blow D, et al. Neuromuscular taping for the upper limb in Cerebral Palsy: A case study in a patient with hemiplegia. Dev. Neurorehabil. 2013; 8423(6):1-4.

54. Butler EE, Ladd AL, Louie Sa, Lamont LE, Wong W, Rose J. Three-dimensional kinematics of the upper limb during a Reach and Grasp Cycle for children. Gait Posture 2010; 32(1):72-77.

55. Turner-Stokes L, Reid K. Three-dimensional motion analysis of upper limb movement in the bowing arm of string-playing musicians. Clin. Biomech. (Bristol, Avon). 1999; 14(6)426-433.

56. Baader AP, Kazennikov O, Wiesendanger M. Coordination of bowing and fingering in violin playing. Brain Res. Cogn. Brain Res. 2005; 23(2-3):436-443.

57. Metcalf CD, Notley SV, Chappell PH, Burridge JH, Yule VT. Validation and application of a computational model for wrist and hand movements using surface markers. IEEE Trans. Biomed. Eng. 2008; 55(3):1199-1210.

58. Cerveri P, De Momi E, Marchente M, Lopomo N, Baud-Bovy G, Barros RML, et al. In vivo validation of a realistic kinematic model for the trapezio-metacarpal joint using an optoelectronic system. Ann. Biomed. Eng. 2008; 36(7):1268-1280.

59. Small CF, Bryant JT, Dwosh IL, Griffiths PM, Pichora DR, Zee B. Validation of a $3 \mathrm{D}$ optoelectronic motion analysis system for the wrist joint. Clin. Biomech. 1996; 11(8): 481-483.

60. Chiu HY, Lin SC, Su FC, Wang ST, Hsu HY. The use of the motion analysis system for evaluation of loss of movement in the finger. J. Hand Surg. Br. 2000; 25(2):195-199.

61. Carpinella I, Jonsdottir J, Ferrarin M. Multi-finger coordination in healthy subjects and stroke patients: a mathematical modelling approach. J. Neuroeng. Rehabil. 2011; 8(1):19. 